\title{
Loss of coherence among coupled oscillators: from defect states to phase turbulence
}

\author{
Yohann Duguet ${ }^{1,2, a)}$ and Yuri L. Maistrenko ${ }^{\dagger 1,2}$ \\ ${ }^{1) \star}$ LIMSI-CNRS, Université Paris Saclay, F-91405 Orsay, France \\ ${ }^{2) \dagger}$ Institute of Mathematics and Centre for Medical and Biotechnical Research, Academy of Sciences of Ukraine, \\ Kyiv, Ukraine
}

(Dated: 21 December 2019)

\begin{abstract}
Synchronisation of a large ensemble of identical phase oscillators with a non-local kernel and a phase lag parameter $\alpha$, is investigated for the classical Kuramoto-Sakaguchi model on a ring. We demonstrate, for low enough coupling radius $r$ and $\alpha$ below $\pi / 2$, a phase transition between coherence and phase turbulence via so-called defect states which arise at the early stage of the transition. The defect states are a novel object resulting from the concatenation of two or more uniformly twisted waves with different wavenumbers. Upon further increase of $\alpha$ defects lose their stability and give rise to spatiotemporal intermittency, resulting eventually in developed phase turbulence. Simulations close to the thermodynamic limit indicate that this phase transition is characterized by non-universal critical exponents.
\end{abstract}

PACS numbers: $05.45 \mathrm{Xt}, 89.75 \mathrm{Fb}$

Keywords: Synchronization; coupled oscillators, Structures and organization in complex systems

When many identical oscillators are coupled together, full synchronisation and rotating waves are not the only possible types of dynamics. Much attention is paid to the evolution from coherent to incoherent behaviour as the parameters are varied, and to its universality. Here we review one of the most studied paradigmatic models of phase oscillators coupled together in a non-local fashion. For this system a nowadays classical scenario has been postulated based on the appearance of special solutions called chimera states. To our surprise, we demonstrate that for smaller coupling ranges, another route towards incoherence is the rule, based on the spatial proliferation of disorder via the interaction of defects ending eventually in spatio-temporal intermittency and phase turbulence. The present route is well described by the statistical concept of continuous phase transition, and features a critical parameter below which no disorder can spread. This finding suggests new bridges between nonlinear network dynamics and statistical physics with prospective applications in various disciplines.

\section{INTRODUCTION}

\section{A. Synchronisation and phase transitions}

The study of large ensembles of self-sustained oscillators coupled together has a deep impact in many diverse

\footnotetext{
a)Electronic mail: duguet@limsi.fr
}

areas of applied physics and other disciplines such as collective animal behaviour, oscillatory chemical reactions, neurons dynamics, Josephson junctions, spermatozoid transport or the stability of pedestrian bridges ${ }^{1}$. The important open questions involve the dynamical and statistical characterisation of the macroscopic states of the system, the transitions between them and the mechanisms responsible for the loss of coherence as the parameters are varied. Since the early theories of Winfree $^{2}$ and Kuramoto ${ }^{3}$, collective behaviour in oscillator arrays is investigated using paradigmatic models. Many such models have considered the interaction between oscillators with different frequencies, where each oscillator is coupled indifferently with all the others. Rather than this mean-field description, we are interested here in coupling kernels whose coupling range is intermediate between local and global, such that each oscillator is coupled to only a finite number of other oscillators. Such situations are found ubiquitously in biology when the coupling between different units depends on their mutual distance and on a threshold value. This is the case when the interaction between individuals relies on visual or auditory stimuli, where the strength of the received stimulus decreases with the distance and vanishes beyond a given distance, as well as in many other fields of Nature and Technology.

The situation where coupling is global corresponds to the classical Kuramoto model ${ }^{4}$ where $N$ phase oscillators with distributed frequencies $\omega_{i}(i=1, \ldots, N)$ are coupled in an all-to-all manner. It is well-known that in one spatial dimension with $N \gg 1$, for a given coupling strength $K$, progressive narrowing of the (unimodal) frequency distribution $\left\{\omega_{i}\right\}$ leads to a continuous phase transition towards synchrony ${ }^{1,3}$. We consider instead in this paper a large number $N$ of identical oscillators, i.e. $\omega_{i}=\omega_{0}$ for all $i$, when no phase transition is expected to occur. The 
generalisation of the Kuramoto model investigated here is the Kuramoto-Sakaguchi (KS) model ${ }^{5}$ : the paradigmatic model on a ring obeys Eq. (1) for indices $i=1, \ldots, N$, where $\phi_{i}(t)$ is the instantaneous phase of the $\mathrm{i}^{t h}$ oscillator defined modulo $2 \pi, R$ defines the coupling radius and $\alpha$ is the phase-lagged parameter:

$$
\dot{\phi}_{i}=\omega_{0}-\frac{K}{2 R+1} \sum_{j=i-R}^{i+R} \sin \left(\phi_{i}-\phi_{j}+\alpha\right) .
$$

Inside the sine coupling function, the coupling between two oscillators $i$ and $j(i \neq j)$ is minimal when their phases differ by an angle $\alpha$, called frustration parameter and expressed in radians. The parameter $\alpha \geq 0$ governs the attraction $(\alpha<\pi / 2)$ or repulsion $(\pi / 2<\alpha<\pi)$ between the oscillators, and we consider below the attractive case with positive $\alpha$ close to $\pi / 2$. For $\alpha=0$, the system derives from a gradient functional, while for $\alpha=\pi / 2$ it is conservative. The control parameter $\alpha$ hence provides a continuous link from the gradient to the integrable regime. Besides in the continuous limit $N \rightarrow \infty$, $r=R / N=$ const, $\alpha \neq 0$ leads to a nonlinear phase equation supporting one-dimensional phase turbulence. Phase turbulence has also been reported in large but finite populations of oscillators ${ }^{6,7}$. This system governs the dynamics of frustrated rotators, with biological applications such as the synchronisation of flagella or of spermatozoid tails ${ }^{8}$. The KS coupling is known to support complex transition scenarios with possible hysteresis ${ }^{5,9}$. The situation where all oscillators have identical frequencies has been less studied as no phase transition has been predicted so far.

It is the goal of this paper to demonstrate that a nonuniversal continuous phase transition occurs at the onset of synchrony of the KS model with identical oscillators. The recent popularity of the non-local system is due to the discovery of chimera states ${ }^{10,11}$, featuring robust spatial coexistence of synchronous and asynchronous zones in network of coupled oscillators (see ${ }^{12-14}$ for recent reviews). In the last decade, starting with Ref. ${ }^{15}$, nonlocally coupled KS system has been extensively studied when the coupling extends, with a constant intensity, to a finite number $2 R$ of the neighbouring oscillators. In this case, parameter regions for multiple chimera states have been identified for non-small values of the coupling radius $r=R / N$ and for large enough values of $\alpha<\pi / 2$ close to $\pi / 2^{16}$. The fate of the chimera states in extended systems as $N$ goes large (with $R$ fixed, so that $r=R / N \ll 1)$, as well as their relevance to the actual dynamics, remains an open question.

We show that for $r=R / N$ small enough the desynchronization transition in the KS model starts with the appearance of so-called defect states. These are novel objects, resulting from the concatenation of two or more uniformly twisted waves with different wavenumbers. Upon further increase of $\alpha$ defects lose their stability giving rise to spatiotemporal intermittency and resulting eventually in developed phase turbulence.
The structure of this paper is as follows : in Section IB, the system under study and the main observables for this investigation are formulated. In Section II, a novel scenario for the transition from coherent dynamics (in the form of twisted waves) to phase turbulence via defect states and spatiotemporal imtermittency, is described. Finally, in Section III the nature of the phase transition is investigated for large $N$, and the corresponding critical exponents are documented. Conclusions are given in Section IV.

\section{B. Simulations of the K-S system and observables}

The system (1) is advanced in time using the $4^{\text {th }}$ order Runge-Kutta algorithm, using a timestep $\Delta t=0.02$. Boundary conditions are periodic and, without lack of generality, $K=1$ and $\omega_{0}=0$. All simulations are initialised with phases $\phi_{i}$ drawn from a uniform distribution in $[0: 2 \pi]$. The complex global order parameter $Z(t)$ is classically defined as

$$
Z(t)=\frac{1}{N} \sum_{j=1}^{N} e^{i \phi_{j}(t)} .
$$

A complex local order parameter is also defined as

$$
z_{l o c}(k, t)=\frac{1}{2 d+1}\left|\sum_{j=k-d}^{k+d} e^{i \phi_{j}(t)}\right|,
$$

where in practice $d=3$, without a strong influence on the results for $R \geq d$. A turbulent fraction, playing the role of a global positive order parameter, can be defined from the thresholding of $z_{l o c}$ by :

$$
F_{t}(t)=\frac{1}{N} \sum_{i=1}^{N} H\left(\theta-z_{l o c}(i, t)\right),
$$

where $H$ is the usual Heaviside function. The threshold $\theta=1 / 2$ has been chosen by trial and error.

\section{TRANSITION FROM COHERENCE TO PHASE TURBULENCE}

Eq. (1) admits many different coexisting regimes even in the attracting range $\alpha \in(0, \pi / 2)$. Besides the simple stable analytical solutions, among which fully synchronous state and twisted waves (called also splay states), new regimes have been identified here by numerical simulation. The huge multistability of the system implies that different initial conditions might lead to different end states. Since we are interested in the loss of coherence in the system (1), the attractors of Eq. (1) are listed and described by increasing complexity as $\alpha$ is increased. The situation is summarised schematically in the stability diagram in fig. 1 computed for $N=8000$. 
The critical line $\alpha_{c}=g(r)$ in Fig. 1 separates the regimes qualified as 'laminar' from 'turbulent'. The laminar regimes include uniformly twisted states and defect states illustrated in Fig. 2 a) and b), respectively. They are characterised by turbulent fraction $F_{t} \approx 0$ whereas the latter, "turbulent" states include all the regimes with non-zero average turbulent fraction $\left\langle F_{t}\right\rangle \neq 0$ including spatiotemporal intermittency (examples in Fig.2c and 2d) and developed phase turbulence (Fig.2e). Representative snapshots in Fig. 2 are obtained along a specific line $R=3$ with an increase of $\alpha$. They demonstrate progressive complication of the system dynamics in the coherence-incoherent transition described below. In Section III we will show how the locus $\alpha_{c}=g(r)$ turns, for constant $R$ into a proper critical point characterised by power-law statistics and a set of critical exponents as $N$ goes to infinity. The current section is devoted to the different regimes already identified close to $\alpha_{c}$ for finite $N$.

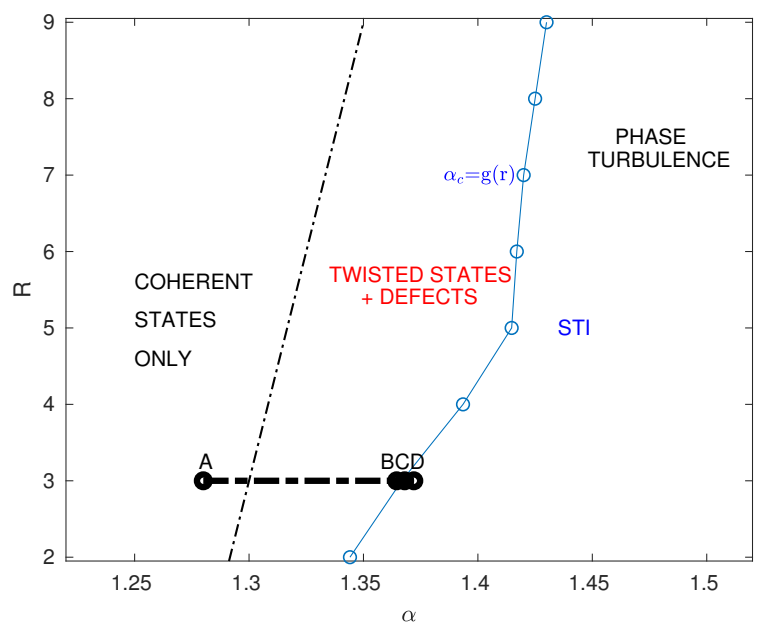

FIG. 1. Stability diagram of the model (1) at the transition from coherent to turbulent phase, etimated for $\mathrm{N}=8000$. The points A,B,C, and D correspond to different regimes displayed in Fig. 2. Blue line : critical line $\alpha_{c}=g(r)$ marking the onset of $F_{t} \neq 0$. Dash-dotted black line: other line marking the complete disappearance of defect states.

\section{A. Synchronised state}

The simplest solution, existing for all parameters $N, R$ and $\alpha$, is the fully synchronised state $\phi_{i}(t)=\omega t+\phi_{0}$ for some value of $\omega$ coinciding with $\omega_{0}$ at $\alpha=0$ and different otherwise. This solution is known to be linearly stable for all $\alpha$ in $[0, \pi / 2)$. It satisfies $|Z|=1$ and thus $F_{t}=0$, and is hence an absorbing state in the language of phase transitions ${ }^{17}$.
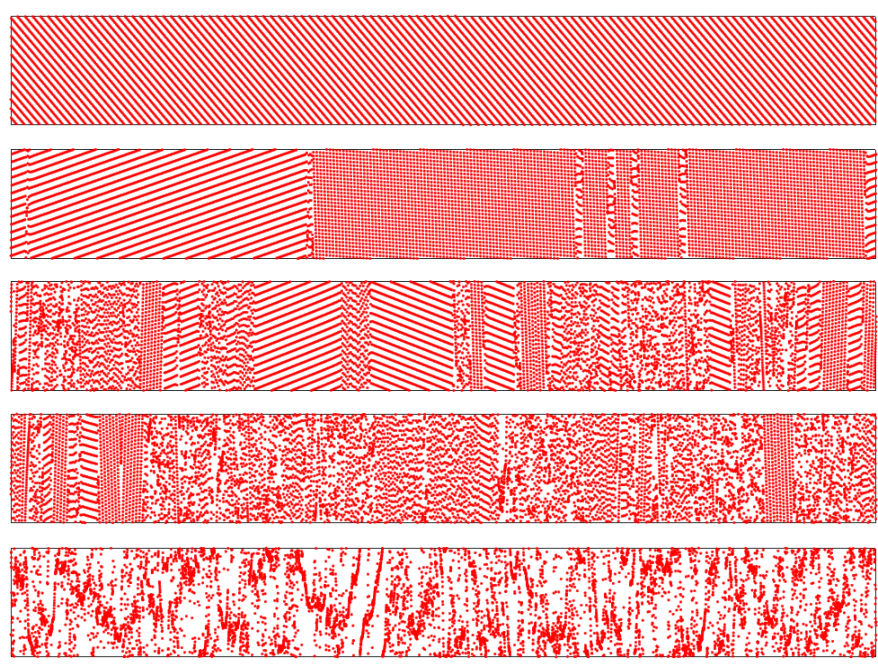

FIG. 2. Progressive complication of the system dynamics at the coherence-incoherent transition along the bold line in Fig. 1. From top to bottom: instantaneous phase $\phi_{i}(k)$ as a function of oscillator position $k$ for points A) $\alpha=1.28$, B) $\alpha=1.3645$, C) $\alpha=1.368$, D) $\alpha=1.372$ and for the point E) $(\alpha=1.48, R=44)$. Labels A,B,C, D correspond to the points displayed in Fig. 1. $\mathrm{N}=8000$, all states $\mathrm{A}-\mathrm{D}$ visualised after a transient of duration $T=4 \times 10^{5}$, state E after $T=10^{3}$.

\section{B. Uniformly twisted waves}

The system (1) admits other simple periodic states in the form of homogeneous travelling waves $\phi_{i}(t)=\omega t+k \frac{2 \pi}{N} i+\phi_{0}$. Here $k$ is a wavenumber which can be any integer between $-(N-1)$ and $N-1$. See Fig. 2 a) for an illustration of $k$-twisted state with $k=-100$ of system (1) with $N=8000$. The fully synchronous state $\phi_{i}(t)=\omega t+\phi_{0}$ can also be referred as a special case of twisted states with $k=0$. It represents the only attractor for $k>1 / 3$ (if one does not count chimera states which exist for $\alpha>1.3$ only and represent, actually, exponentially long transients ${ }^{18}$. On the other hand, for $r<1 / 3$ synchronous state competes in the sense of stability with $k$-twisted states, each of which is stable at $r<1 / 3 k^{19}$. Solutions with $k \neq 0$ satisfy $|Z|=0$ and $1 / 2 \ll z_{l o c}<1$, hence they also satisfy $F_{t}=0$. The stability result in Ref. ${ }^{19}$ was obtained for $\alpha=0$. Besides, as can be shown, linear stability of such twisted states carries over to all positive values of $\alpha$ up to $\pi / 2^{20}$. Therefore, they all are co-existing attractors as $r$ decreases. Note that linear stability of a given state tells nothing about the size of its attraction basin, hence about its likelihood to be identified in simulations from arbitrary initial conditions. 


\section{Defect states}

Among the variety of regimes characteristic to the non-locally coupled KS system (1) we find a novel class of solutions, so-called defect states in which the whole medium is splitted into two or more domains with different twisted states in each of them, see an example in Fig.2b. The concatenation of different twisted states with different $k$ 's is reminiscent of grain boundaries in polycristalline textures, and the interfaces are interpreted as defects $^{21}$. Each interface between two neighbouring subdomains typically extends over a small number $O(R)$ of oscillators, and appears narrow in comparison with the total spatial extent given by $N$. Spatial periodicity imposes the number of such interfaces to be at least two. The example in Fig. 2b with $N=8000$ features for two such interfaces separating two twisted states with wavenumbers $k \approx 500$ and $k \approx-100$. The left interface behaves like a sink where two travelling waves on either side propagate in converging directions and collide (near $i \approx 3600$ in fig. $2 \mathrm{~b}$ ). The sinks alternates with a source out of which travelling waves radiate (near $i \approx 6200$ ).

In our simulations with random initial conditions, we observed many defect states with different numbers of interfaces and different $k$-twisted travelling waves inside the domains. Defects travel as a whole, depending on the values of $k$ on either side. Interfaces between two subdomains characterised by exactly opposite values of $k$, however, do not propagate. The long-time dynamics of defects remains an open question. In many instances they were observed to persist during the entire observation simulations (as long as $10^{6}$ time units for the longest runs), and hence behave as effective attractors. In other cases, they appear as metastable objects with finite lifetimes. Fig.1 shows schematically the parameter region for the defect states found in our simulations.

To our knowledge, this kind behavior manifested by defect states has never been reported for KS system (1). A deeper investigation of these new localised coherent structures, their stability, as well as their basin structure is called for. For $R=3$, defects have been tracked down to as low as $\alpha \approx 1.30$, see Fig. 1 . For lower $\alpha$ the defect lifetimes becomes shorter than the observation time, they are seen to decay slowly, leaving the stage after a long reorganisation to one of the $k$-twisted states (possibly with $k=0$ ). For larger $\alpha$, defect states emerge naturally as end results of transient simulations of the other, disordered regime called spatio-temporal intermittency, see a characteristic example in Fig. 3 (right).

\section{Spatiotemporal intermittency}

Upon further increase of $\alpha$, asynchronous dynamics arises characterised by low values of $z_{l o c}$ amidst local intermittent excursions of $z_{l o c}$ above $1 / 2$. As a conse- quence, the turbulent fraction $F_{t}$ stays strictly below $100 \%$ as a signature of spatial coexistence between synchronous and asynchronous motion, as illustrated by the phase snapshots in Figs. 2(c) and (d). As long as the disordered dynamics is active, the value of $F_{t}$ fluctuates around a non-zero mean, in contrast with $F_{t}=0$ for the synchronised state, for the twisted states and for the defect states. Borrowing from the hydrodynamics literature $^{22}$, we label as laminar all zones with $z_{l o c}<1 / 2$, and the complementary zones are labelled as turbulent. The laminar zones are not strictly inactive, as they support the leftwise or rightwise propagation of narrow regions with locally low $z_{l o c}$, akin to defects. This is most evident in Fig. 3 amidst the yellow regions. Two generic
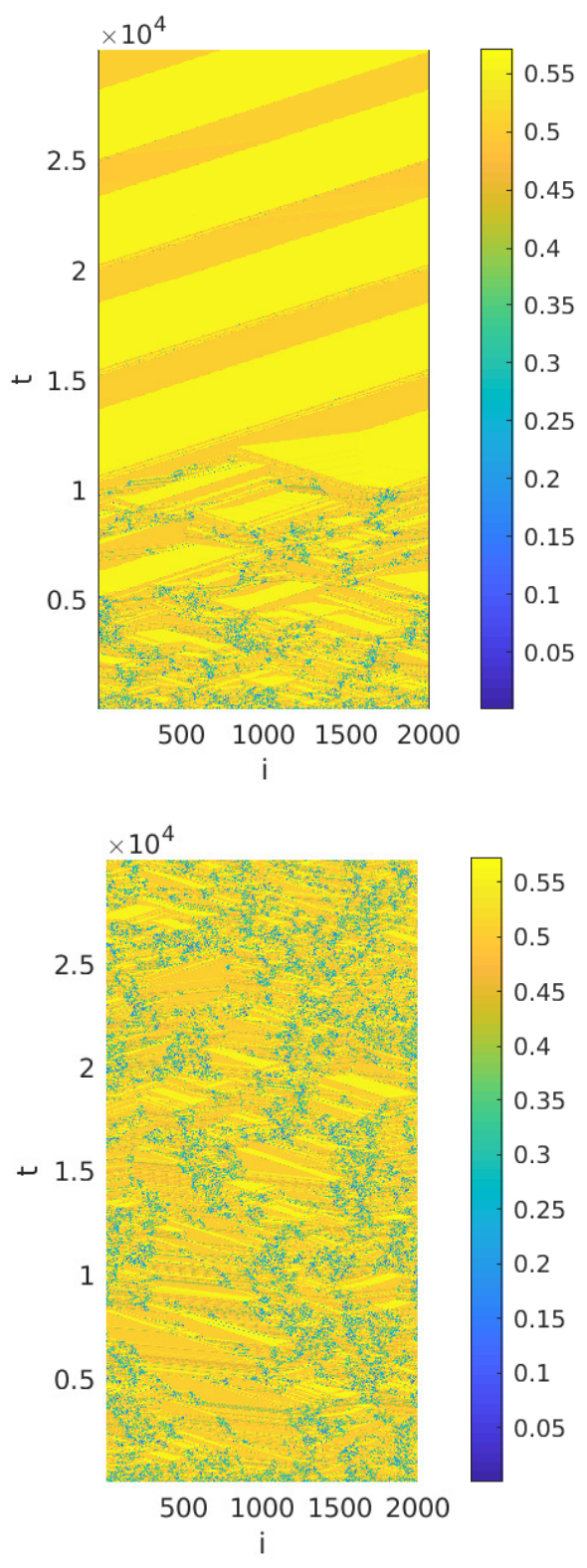

FIG. 3. Space-time diagrams of $z_{l o c}(i, t), \mathrm{N}=2000, \mathrm{R}=3$ : top) transient STI, $\alpha=1.365$, bottom) sustained STI, $\alpha=1.372$. 
cases are illustrated in Fig. 3 for $R=3$ : either the lifetime is finite and disorder is transient (left) or it is sustained (right). In the transient case (left), only a defect state remains for $t \geq 1.2 \times 10^{4}$ and it persists for the whole simulation time. Two defects separate large zones characterised each by a single twisted state. The interfaces separating different twisted states (i.e. the defects themselves) travel as a consequence of that mismatch between the wavenumbers on their respective left and right. In the sustained case (right), intermittent dynamics of multiply co-existing laminar and turbulent gaps persists for the whole simulation time. Instantaneous snapshots of the phases $\phi_{i}(i, t)$ and of the local order parameter $z_{l o c}(i, t)$ are displayed in fig. 4 in order to highlight the differences between the end states in the transient and sustained STI cases.

For N sufficiently large, we have computed the cumulative distribution of laminar gap sizes in the sustained case, i.e. the probability $P\left(l_{l a m}>L\right)$ that a simply connected laminar hole of size $l_{l a m}$ exceeds a given size $L$. For most parameters away from criticality the distributions are exponential, as obtained in fig. 6. This behavior hence qualifies as a sustained spatio-temporal intermittency (STI) regime ${ }^{23}$. Importantly, it is different from chimera states as the co-existing multiple laminar and turbulent zones in STI regime are not localized but rather move chaotically, colliding and re-appear.
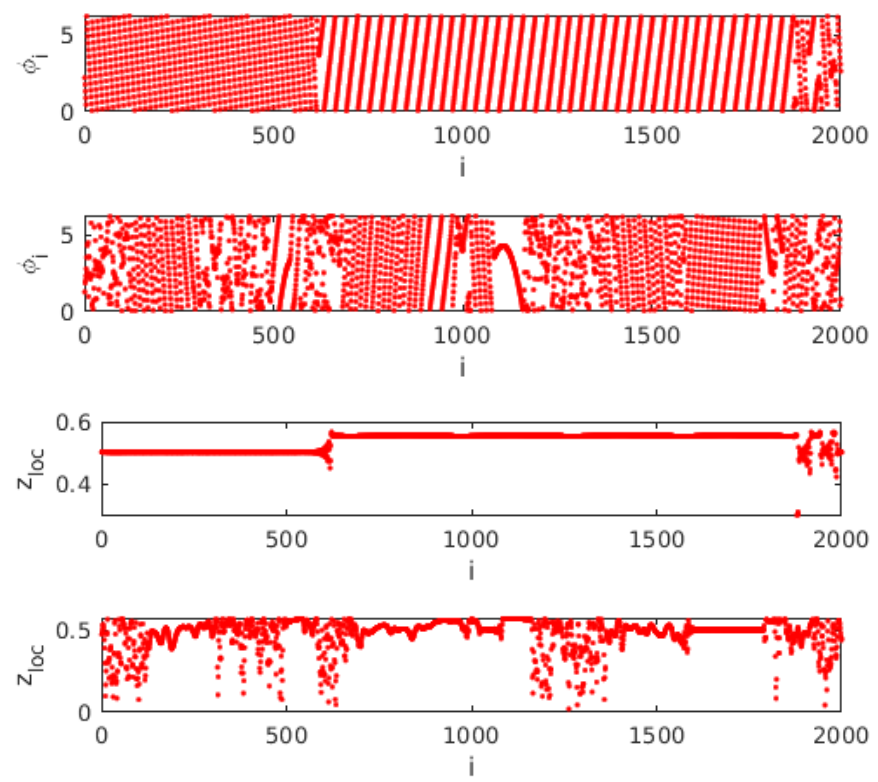

FIG. 4. Instantaneous snapshots corresponding to Figure 3 for $N=2000, \mathrm{R}=3$ at $t=2 \times 10^{4}$. From top to bottom : phases for $\alpha=1.365$ and 1.372 , local order parameter $z_{l o c}$ for the same values of $\alpha$.

A time-dependent quantity $n(t)$, interpretable as a mean number of "active" oscillators, is defined as

$$
n(t)=\sum_{k=1}^{N} H\left(R_{d}(k, t)-\theta^{\prime}\right),
$$

where $R_{d}(k, t)=\left|z_{l o c}(k, t)-z_{l o c}(k+1, t)\right|$ and $\theta^{\prime}=0.05$. $n$ measures the numbers of oscillators involved belonging to defect states at a given time. $n(t)$ close to criticality is displayed in fig. 5. Two main regimes emerge, both with temporal flucutuations of $n$, emerge : above $\alpha_{c}, n(t)$ saturates to a steady regime with $n \gg 1$, whereas close to or $\alpha_{c} n$ decreases and then saturates to an $O(1)$ number.

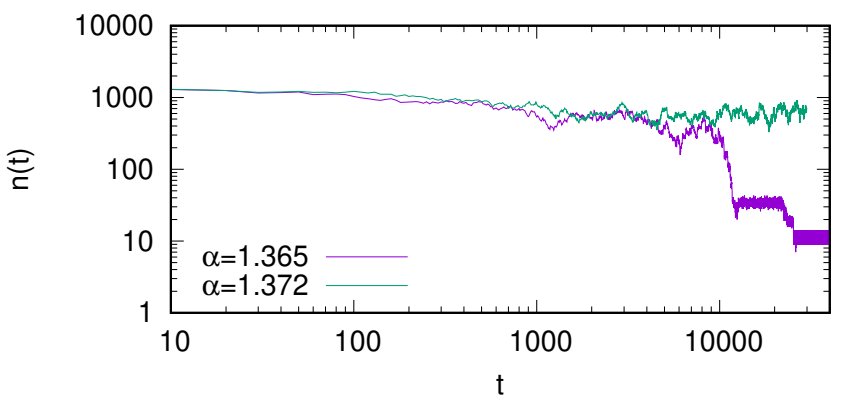

FIG. 5. Number of oscillators $n(t)$ involved in defects, $\mathrm{R}=3$, $N=2000, \theta=0.05$ same values of $\alpha$ as Fig. 4, log-log plot.

Our simulations confirm that the transition between transient and sustained STI occurs along a critical line $\alpha_{c}=g(r)$ displayed in Fig. 1 for $N=2000$. Above the threshold value $\alpha_{c}=g(r)$ located strictly between 1.30 and $\pi / 2$, the statistical equilibrium regimes reached correspond to sustained STI, whereas for $\alpha<\alpha_{c}$ STI regimes are only transient, resulting eventually in a defect state or in a regular twisted or synchronous state. An accurate estimation of $\alpha_{c}$ in the large $N$ limit constitutes the main part of the next Section III.

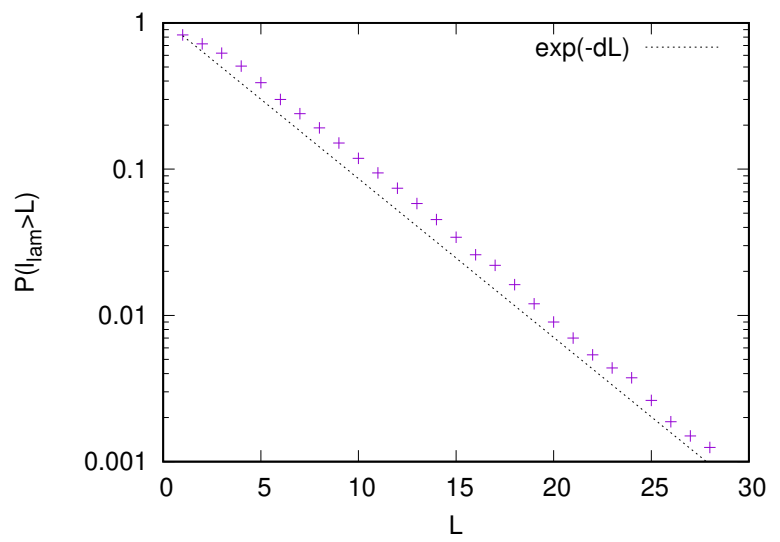

FIG. 6. Statistics of laminar gap distribution $P\left(l_{\text {lam }}>L\right)$ in the STI regime for $\mathrm{N}=9 \times 10^{4}, \mathrm{R}=3, \alpha=1.43$, logarithmic scale. Fit by exponential function $e^{-d L}$ with $d=0.25$. 


\section{E. Phase turbulence regime}

Upon increasing the value of $\alpha$ and decreasing $r$, the turbulent fraction of the STI regime increases until no laminar zones are left in the space. For small enough but non-vanishing $r$ down to at least $\approx O\left(10^{-3}\right), z_{\text {loc }}$ remains below $1 / 2$ everywhere, so that $F_{t} \approx 1$ at all times. As a consequence, asynchrony between neighbouring oscillators is now the rule. This type of space-time dynamics corresponds to phase turbulence ${ }^{7}$. It is illustrated in Fig. 7 for $N=8000, R=44\left(r=5.5 \times 10^{-3}\right)$ and $\alpha=1.48$; a snapshot is shown in Fig.2e. The cross-over from STI with high turbulent fraction to phase turbulence is gradual.

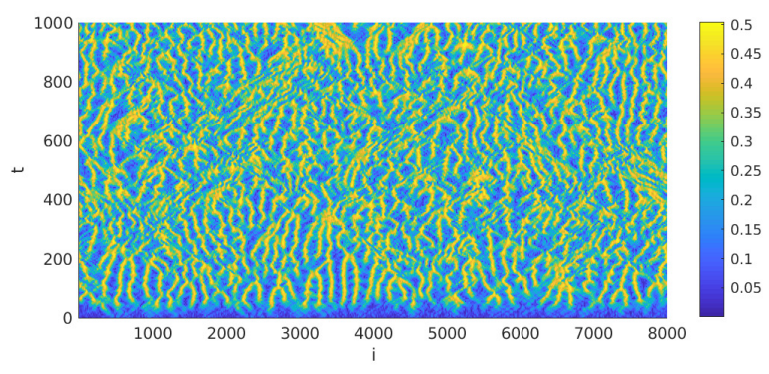

FIG. 7. Example of phase turbulence regime for $N=8000$, $R=44 \quad(r=44 / 8000)$ and $\alpha=1.48$. Space-time diagram for $z_{l o c}(i, t)$.

\section{F. Lack of relevance of chimera states}

Chimera states ${ }^{10,11}$ constitutes another class of longtime dynamics in the non-locally coupled KS model. As documented in ${ }^{16}$, for system (1) multi-head chimera states exist in extended parameter regions attached to $\alpha=\pi / 2$, but mostly at non-small coupling radius $r=$ $R / N$. In Fig.1 from ${ }^{16}$ the detailed structure of the $n_{H^{-}}$ head chimera regions is shown for $n_{H}$ from 1 to 10 . The chimeras compete in the phase space with the fully synchronous state and with the $k$-twisted states stabilized by the decrease of $r$ beyond $1 / 3^{19}$, but not with the defect and STI regimes as they arise at sufficiently smaller values of $r$. Note, that there is visual self-similarity of the $n_{H}$-head chimera regions in the sense that $R_{s n}$ decreases apparently as $O\left(n_{H}^{-1}\right)$, possibly intersecting the STI regions for large $n_{H}$. However, no such chimera state has emerged spontaneously in our simulations for $r<0.1$. This suggests that the multi-head chimeras have irrelevantly small basins of attraction. Instead only the STI regime were found as $r$ decreases and $\alpha$ becomes closer to $\pi / 2$.

For the KS model with exponential kernel $G(x)=p^{-1} e^{-p|x|}$, only classical one-head chimeras exist (for finite effective coupling radius $r=1 / p$ ), as the multi-headed chimeras appears to be unstable ${ }^{16,24}$. The existence of the STI regime, on the other hand, has been confirmed in our simulations using the present code with an exponential kernel as well. We conclude that regardless of the exact coupling kernel, for low effective coupling radius $r$ chimera states are not as typical as spatiotemporal intermittency regimes (although their existence and their stability can not be excluded depending on the form of kernel).

\section{CONTINUOUS PHASE TRANSITION}

Two distinct limits $N \rightarrow \infty$ can be considered in discrete oscillator arrays. In the continuous limit, the spatial density of oscillators diverges while the normalised coupling range $r=R / N$ is held constant. An a priori different limit, directly relevant to the study of phase transitions, is the thermodynamic limit, where both $N$ and $r^{-1}=N / R$ increase to infinity, while $R$ is fixed. Considering the thermodynamic limit corresponds to increasing the domain size while maintaining a constant spatial density of oscillators. Both limits are indistinguishable in the case of all-to-all coupling due to the absence of metrics. In this section we investigate the behaviour of the $\mathrm{KS}$ system for a finite but large number $N$ of identical oscillators at small $r$ and over very long observation times $T$, i.e. close to the true thermodynamic limit. The onset of asynchrony corresponds to a continuous phase transition characterised by a well-defined critical line $g_{\infty}(\alpha, r)=0$ in the $(\alpha, r)$ space. In practice the diverging lengthscales and timescales close to the critical line make the simulations very costly. This is taken care of by using sharedmemory Open MP with $N=9 \times 10^{4}$ and $T=10^{6}$. In the numerical exploration only $\alpha$ is varied for fixed $R=3$.

\section{A. Statistics close to the thermodynamic limit}

We first monitor the dependence of the equilibrium turbulent fraction $\left\langle F_{t}\right\rangle$ as a function of the distance $\varepsilon$ to the critical point, i.e. $\varepsilon=\left(\alpha-\alpha_{c}\right) / \alpha_{c}$. This is displayed in fig. 8 , where $\alpha_{c}$ has been estimated by trial and error in order to make the algebraic scaling as evident as possible. A fit of the form $\left\langle F_{t}\right\rangle \sim\left(\alpha-\alpha_{c}\right)^{\beta}$ is found here for $\alpha_{c} \approx 1.3643$. The algebraic scaling of $\left\langle F_{t}\right\rangle$ for $\alpha$ closest to $\alpha_{c}$ is valid over more than a decade down to $\left\langle F_{t}\right\rangle \approx 10^{-2}$. The power-law exponent is estimated as $\beta=0.78 \pm 0.01$. The values of $\alpha_{c}$ for $\mathrm{R}=3$ for increasing $\mathrm{N}$ in Table I suggest that the statistics are converged.

Another critical exponent, noted here $a$, occurs in the temporal decay of the turbulent fraction when simulations are initialised by random fields with $F_{t}(t=$ $0)=O(1)$. Although the algebraic decay is evident, fitting an exponent from fig. 9 is not easy in practice. An accurate value of $a$ was found instead by plotting the scaling function $f$ appearing in the relation $t^{a} F_{t}(t) \sim f\left(\varepsilon^{\nu_{\|}} t\right)$ 


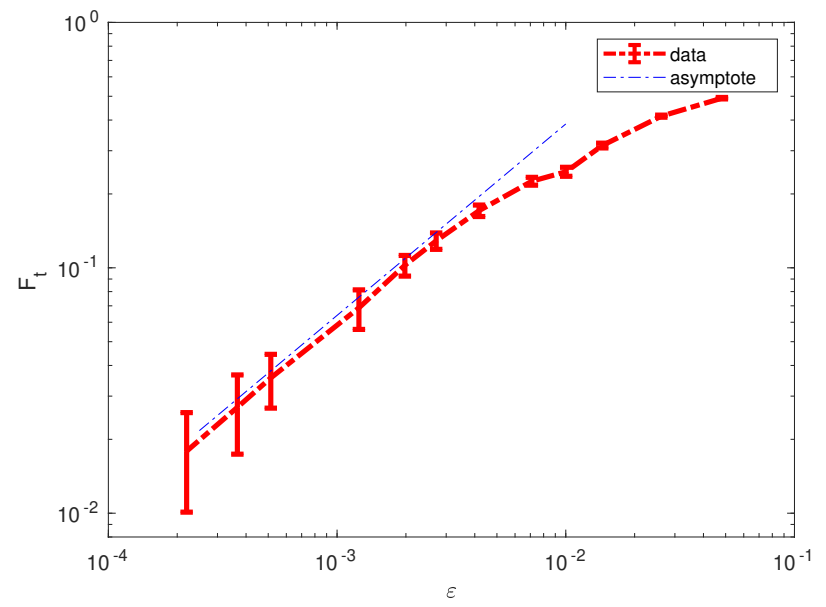

FIG. 8. Equilibrium turbulent fraction $F_{t}(\alpha)$ for $\mathrm{N}=9 \times 10^{4}$ and $\mathrm{R}=3$ (red crosses) with errorbars, as a function of the distance to threshold $\varepsilon=\frac{\alpha-\alpha_{c}}{\alpha_{c}}$. Asymptotic fit $C \varepsilon^{\beta}$ with $C=12$ and $\beta=0.78$ (blue dash-dotted line).

\begin{tabular}{|c|c|c|}
\hline$N$ & $\alpha_{c}$ & $\delta \alpha_{c}$ \\
\hline \hline $2 \times 10^{3}$ & 1.375 & 0.005 \\
$8 \times 10^{3}$ & 1.3667 & 0.0003 \\
$9 \times 10^{4}$ & 1.3643 & 0.0002 \\
\hline
\end{tabular}

TABLE I. Critical value $\alpha_{c}$ (with error margin $\delta \alpha_{c}$ ) for $\mathrm{N}=3$ depending on the value of $\mathrm{N}$

and adjusting the exponents until all curves in fig. 5 collapse. This leads to an estimation of $a=0.40 \pm 0.05$. The value of $\alpha$ where this fit holds best is consistent with the value of $\alpha_{c} \approx 1.3643$.

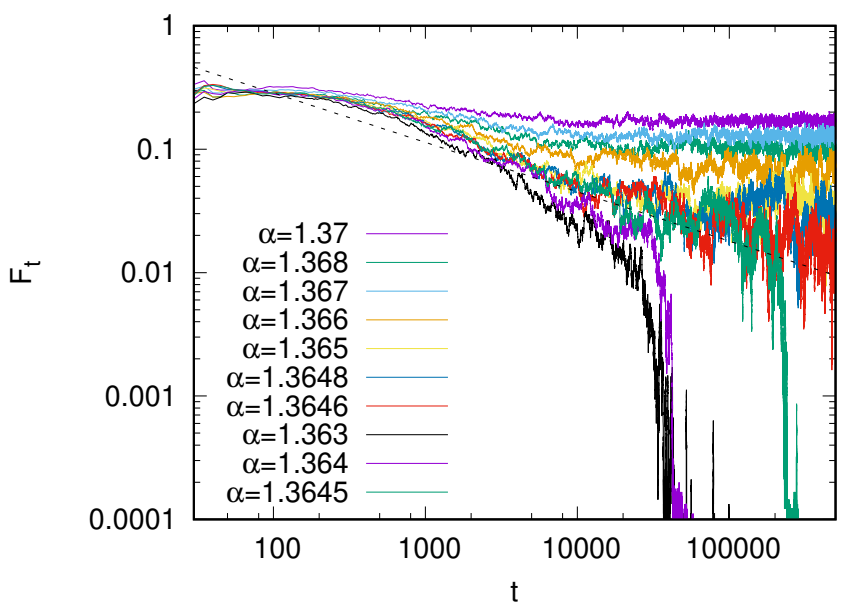

FIG. 9. Instantaneous turbulent fraction $F_{t}$ versus time for $\mathrm{N}=9 \times 10^{4}, \mathrm{R}=3$ and varying $\alpha$. Dashed thick line : algebraic decay $O\left(t^{-0.4}\right)$.

The cumulative distribution of laminar gap sizes

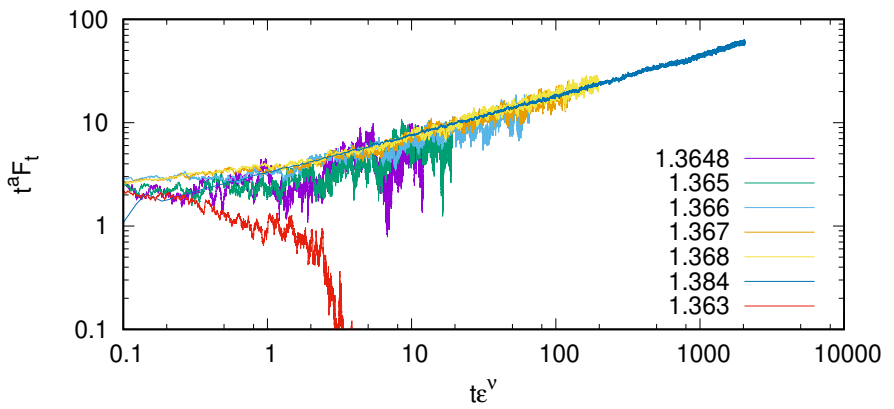

FIG. 10. Scaling function for $\mathrm{N}=9 \times 10^{-4}, \mathrm{R}=3$, various $\alpha$.

$P\left(l_{\text {lam }}>L\right)$ has been recomputed for $\alpha$ closer to $\alpha_{c}$. Whereas away from criticality the distribution is clearly exponential (see fig. 6), fig. 11 displays a cross-over from exponential to algebraic decay $P\left(l_{\text {lam }}>L\right) \sim L^{-\mu_{\perp}}$. The best fit, valid over more than two decades, is again consistent with the choice of $\alpha_{c}$. It yields a third independent critical exponent $\mu_{\perp}=0.56 \pm 0.02$.

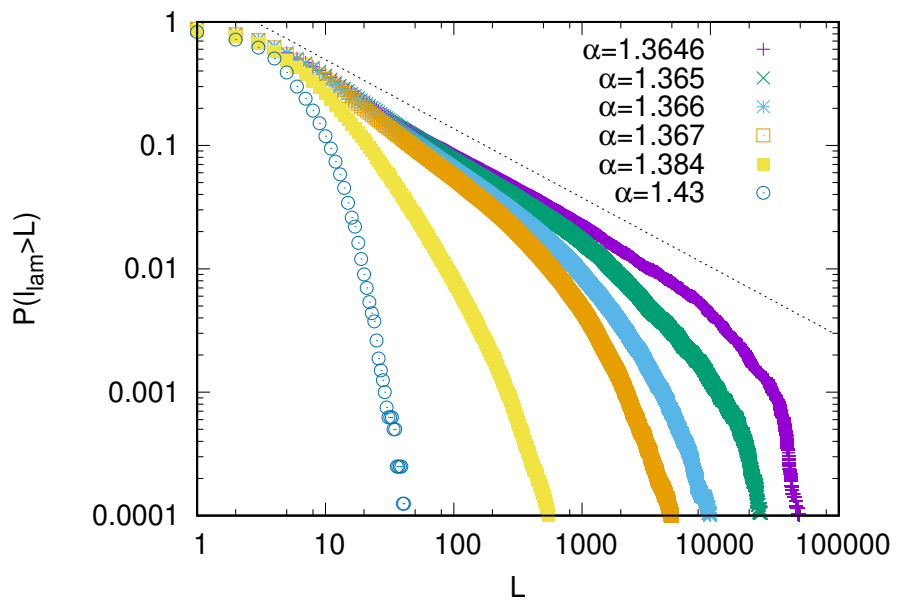

FIG. 11. Statistics of laminar gap distribution for $\mathrm{N}=9 \times$ $10^{-4}, \mathrm{R}=3$ for various $\alpha$. Thin dotted line : fit $O\left(L^{-0.56}\right)$.

\section{B. Breakdown of universality}

These statistics are consistent with a continuous phase transition occuring for vanishing $r$ at $\alpha_{c} \approx 1.364$. The three critical exponents $\beta=0.78, a=0.40$ and $\mu_{\perp}=0.56$ do not belong to any known universality class. In particular, whereas the visual aspect of the space-time diagrams, such as the transient in fig. 3(left), is reminiscent of contact processes such as $(1+1)$-D directed percolation $(\mathrm{DP})^{22}$, this hypothesis is not supported at all by the calculation of the critical exponents above (see Table II). Even on the qualitative level there are clear discrepancies with the DP picture in one spatial dimension, due to violations of the hypothesis behind the Janssen-Grassberger 


\begin{tabular}{|c|c|c|}
\hline & $D P$ & $K S$ \\
\hline \hline$\beta$ & 0.276 & 0.78 \\
$a$ & 0.159 & 0.40 \\
$\mu_{\perp}$ & 1.75 & 0.56 \\
\hline
\end{tabular}

TABLE II. Critical exponents for $(1+1)$-DP (left column) and for the KS system with $R=3$ (right column, $\mathrm{N}=9 \times 10^{4}$ ).

conjecture $^{25,26}$. This conjecture states that (1+1)-D DP is guaranteed if the system features a unique fluctuationfree absorbing state in competition with a fluctuating phase, a positive one-component scalar order parameter, short-range interactions and no additional symmetries. Although the interactions are non-local, the finite coupling range and the limit $R / N \rightarrow 0$ fulfil the short-range interaction hypothesis. Yet the first hypothesis, namely the uniqueness of the absorbing state, is here violated for several reasons. First, trivial absorbing states are not unique as there are multiple stable twisted states with different wavenumbers. In the vanishing $R / N$ limit, this corresponds to a countable infinity of absorbing states. Besides, the robustness in time of the defect solutions makes them interpretable in practice to a different type of absorbing states, which unlike the twisted states do feature temporal fluctuations. A similar situation was identified earlier for coupled map lattices with diffusive coupling and synchronous updating ${ }^{23,27}$. In the corresponding systems, the multitude of absorbing states also leads to propagating defects, forming for high densities a 'soliton gas'28. The existence and structure of the defects originates from the spatial coexistence of different twisted states in their stable parameter region. This situation was modelled by Bohr and co-workers, using a tuneable density of defects in a probabilistic cellular automaton, which in the absence of defects would belong to the DP class $^{29,30}$. Defects propagating in opposite directions and/or at different speeds have the possibility of colliding, of igniting or reviving turbulent fluctuations, of modifying the long-time statistics and the critical exponents, and thus of driving the system out of the DP class. The propagation of defects, visible in fig. 3 fully supports the adequacy of Bohr's model to the present system. Depending on the parameters, Bohr's model can feature either a non-universal continuous transition or a discontinuous one. In the present study of the Kuramoto-Sakaguchi system, the analogy suggests that the exponents calculated for the present type of kernel and coupling function do not define a novel universality class either, and are thus parameter-dependent even in the thermodynamic limit. A recent study $\left({ }^{31}\right)$ supports our findings about non-universal critical exponents.

\section{CONCLUSIONS}

As a summary, we have identified a novel scenario for the coherence-incoherence transition in spatially ex- tended Kuramoto-Sakaguchi model with non-local coupling. It does not involve chimera states, as they arise in other parts of the parameter space for larger $r$. This transition is identified for small-to-vanishing values of the coupling range and start with the appearance of the defect states, which then lose stability, giving rise to spatiotemporal intermittency. It carries over towards the thermodynamic limit where it becomes a continuous phase transition. Unlike the known phase transitions in oscillators arrays ${ }^{1}$ it occurs here in the presence of identical oscillators.

The defect states are novel objects for the KS model. These are spatially solitary structures with deterministic dynamics and long (possibly infinite) lifetimes, propagating at constant velocity. The exact range of their stability is difficult to investigate because of the many possible combinations of twisted waves composing a given defect state, and the impossibility of predicting the final defect structure from arbitrary initial conditions. It deserves future work in relation with the recent discovery of solitary states specific to oscillators networks ${ }^{32}$. Beyond the regime of solitary defects, the yet unreported regime of spatio-temporal intermittency (STI) emerges with a monotonically increasing equilibrium fraction of asynchronicity. Further beyond the STI regime, phase turbulence takes place as anticipated in the early works by Kuramoto ${ }^{5,6}$.

The onset of the complex regime, or conversely the onset of synchrony, occurs across a smooth critical line in the $(\alpha, r)$-parameter plane and requires the statistical framework of phase transitions in the thermodynamic limit. Compared to relatively classical instances where direct percolation is the rule, the situation is made more complicated by the multitude of absorbing states and the long-lasting presence of defects. The consequence is a non-universal set of critical exponents quantitatively different from those of $\mathrm{DP}^{29}$. Evidence for this new coherence-incoherence scenario in the KuramotoSakaguchi system with identical frustrated oscillators calls for a possible generalisation to other types of kernels or coupling functions ${ }^{33}$, different network topologies ${ }^{34}$, noise ${ }^{35}$, time delay ${ }^{36}$, inertia ${ }^{32,37}$, and higher spatial dimensions ${ }^{38-42}$. In particular, the dynamics of defects in higher dimension becomes more complex, and the computational cost would call for reduced order modelling (see e.g. Ref. ${ }^{43}$ ) and specific statistical analysis tools ${ }^{44}$. When this paper was ready for submission we got aware of an arxiv submission ${ }^{31}$ where some questions similar to our Ch. III are discussed.

\section{A. Acknowledgements}

The authors thank P. Manneville, M. Hasler, G. Lemoult, A. Pikovsky, O. Omel'chenko, M. Wolfrum for interesting discussions, as well as SFB-910 and TU-Berlin for travel support. L. Oteski is gratefully acknowledged 
for optimising the simulation code.

${ }^{1}$ A. Pikovsky, M. Rosenblum, and J. Kurths, Synchronisation : A Universal Concept in Nonlinear Sciences (Cambridge University Press, 2001).

${ }^{2} \mathrm{~A}$. T. Winfree, The geometry of biological time (Vol. 12) (Springer, New York, 2001).

${ }^{3}$ Y. Kuramoto, Chemical Oscillations, Waves, and Turbulence (Springer, Berlin, Heidelberg, 1984).

${ }^{4}$ Y. Kuramoto, Self-entrainment of a population of coupled nonlinear oscillators (Springer, Berlin, Germany, 1975).

${ }^{5}$ H. Sakaguchi and Y. Kuramoto, Progress of Theoretical Physics 76, 576 (1986).

${ }^{6}$ Y. Kuramoto, Progress of Theoretical Physics 79, 223 (1984).

${ }^{7}$ Y. Kawamura, Phys. Rev. E 89, 010901 (2014).

${ }^{8}$ N. Uchida and R. Golestanian, Phys. Rev. Lett. 104, 178103 (2010).

${ }^{9}$ E. Omel'chenko and M. Wolfrum, Phys. Rev. Lett. 109, 164101 (2012).

${ }^{10} \mathrm{Y}$. Kuramoto and D. Battogtokh, arXiv preprint condmat/0210694 (2002).

${ }^{11}$ D. M. Abrams and S. H. Strogatz, Phys. Rev. Lett. 93, 174102 (2004).

${ }^{12}$ M. J. Panaggio and D. M. Abrams, Nonlinearity 28, 67 (2015).

${ }^{13}$ E. Schoell, The European Physical Journal Special Topics 225, 891 (2016).

${ }^{14}$ O. Omel'chenko, Nonlinearity 31 (2018).

${ }^{15}$ O. E. Omel'chenko, M. Wolfrum, and Y. L. Maistrenko, Phys. Rev. E 81, 065201 (2010).

${ }^{16}$ Y. L. Maistrenko, A. Vasylenko, O. Sudakov, R. Levchenko, and V. L. Maistrenko, International Journal of Bifurcation and Chaos 24, 1440014 (2014).

${ }^{17}$ M. Henkel, H. Hinrichsen, S. Lübeck, and M. Pleimling, Nonequilibrium phase transitions, vol. 1 (Springer, 2008).

${ }^{18} \mathrm{M}$. Wolfrum and O. Omel'chenko, Phys. Rev. E 84, 015201 (2011).

${ }^{19}$ D. A. Wiley, S. H. Strogatz, and M. Girvan, Chaos 16, 015103 (2006).

${ }^{20} \mathrm{~T}$. Gyrnik, private communication (2014).

${ }^{21}$ M. C. Cross and P. C. Hohenberg, Reviews of Modern Physics 65, 851 (1993).

${ }^{22}$ G. Lemoult, L. Shi, K. Avila, S. V. Jalikop, M. Avila, and B. Hof, Nature Physics (2016).

${ }^{23}$ H. Chaté and P. Manneville, Phys. Rev. Lett. 58, 112 (1987).

${ }^{24}$ L. Smirnov, G. Osipov, and A. Pikovsky, Journal of Physics A: Mathematical and Theoretical 50, 08LT01 (2017).

${ }^{25}$ H. Janssen, Z. f. Physik B 42, 151 (1981).

${ }^{26}$ P. Grassberger, Z. f. Physik B 47, 365 (1982).

${ }^{27}$ G. Rousseau, Ph.D. thesis, Université Paris-Diderot-Paris VII (1998).

${ }^{28}$ P. Grassberger and T. Schreiber, Physica D 50, 177 (1991).

${ }^{29}$ T. Bohr, M. van Hecke, R. Mikkelsen, and M. Ipsen, Phys. Rev. Lett. 86, 5482 (2001).

${ }^{30}$ T. Bohr, M. H. Jensen, G. Paladin, and A. Vulpiani, Dynamical systems approach to turbulence (Cambridge University Press, 2005).

${ }^{31}$ K. Kawase and N. Uchida, arXiv:1907.07285 (2019).

${ }^{32}$ P. Jaros, S. Brezetsky, R. Levchenko, D. Dudkowski, T. Kapitaniak, and Y. Maistrenko, Chaos 28, 011103 (2018).

${ }^{33}$ P. Pikovsky and M. Rosenblum, Physica D 238, 27 (2009).

${ }^{34}$ R. Toenjes, N. Masuda, and H. Kori, Chaos 20, 033108 (2010).

${ }^{35}$ Y. Kawamura, H. Nakao, and Y. Kuramoto, Phys. Rev. E 75, 036209 (2007).

${ }^{36}$ L. Larger, B. Penkovsky, and Y. Maistrenko, Phys. Rev. Lett. 111, 054103 (2013).

${ }^{37}$ S. Olmi, A. Navas, S. Boccaletti, and A. Torcini, Phys. Rev. E 90, 16 (2014).

${ }^{38}$ O. E. Omel'chenko, M. Wolfrum, S. Yanchuk, Y. L. Maistrenko, and O. Sudakov, Phys. Rev. E 85, 036210 (2012).
${ }^{39}$ M. J. Panaggio and D. M. Abrams, Physical review letters 110, 094102 (2013).

${ }^{40}$ J. Xie, E. Knobloch, and H.-C. Kao, Physical Review E 92, 042921 (2015).

${ }^{41}$ Y. Maistrenko, O. Sudakov, O. Osiv, and V. Maistrenko, New Journal of Physics 17, 073037 (2015).

${ }^{42}$ H. W. Lau and J. Davidsen, Phys. Rev. E 94, 010204 (2016).

${ }^{43}$ C. Brito, I. Aranson, and H. Chaté, Phys. Rev. Lett. 90, 068301 (2003).

${ }^{44}$ G. St-Yves and J. Davidsen, J. Chem. Phys. 133, 044909 (2010).

${ }^{1}$ A. Pikovsky, M. Rosenblum, and J. Kurths, Synchronisation : A Universal Concept in Nonlinear Sciences (Cambridge University Press, 2001).

${ }^{2} \mathrm{~A}$. T. Winfree, The geometry of biological time (Vol. 12) (Springer, New York, 2001).

${ }^{3}$ Y. Kuramoto, Chemical Oscillations, Waves, and Turbulence (Springer, Berlin, Heidelberg, 1984).

${ }^{4}$ Y. Kuramoto, Self-entrainment of a population of coupled nonlinear oscillators (Springer, Berlin, Germany, 1975).

${ }^{5}$ H. Sakaguchi and Y. Kuramoto, Progress of Theoretical Physics 76, 576 (1986).

${ }^{6}$ Y. Kuramoto, Progress of Theoretical Physics 79, 223 (1984).

${ }^{7}$ Y. Kawamura, Phys. Rev. E 89, 010901 (2014).

${ }^{8}$ N. Uchida and R. Golestanian, Phys. Rev. Lett. 104, 178103 (2010).

${ }^{9}$ E. Omel'chenko and M. Wolfrum, Phys. Rev. Lett. 109, 164101 (2012).

${ }^{10} \mathrm{Y}$. Kuramoto and D. Battogtokh, arXiv preprint condmat/0210694 (2002).

${ }^{11}$ D. M. Abrams and S. H. Strogatz, Phys. Rev. Lett. 93, 174102 (2004).

12 M. J. Panaggio and D. M. Abrams, Nonlinearity 28, 67 (2015).

${ }^{13}$ E. Schoell, The European Physical Journal Special Topics 225, 891 (2016).

${ }^{14}$ O. Omel'chenko, Nonlinearity 31 (2018).

${ }^{15}$ O. E. Omel'chenko, M. Wolfrum, and Y. L. Maistrenko, Phys. Rev. E 81, 065201 (2010).

${ }^{16}$ Y. L. Maistrenko, A. Vasylenko, O. Sudakov, R. Levchenko, and V. L. Maistrenko, International Journal of Bifurcation and Chaos 24, 1440014 (2014).

${ }^{17}$ M. Henkel, H. Hinrichsen, S. Lübeck, and M. Pleimling, Nonequilibrium phase transitions, vol. 1 (Springer, 2008).

${ }^{18}$ M. Wolfrum and O. Omel'chenko, Phys. Rev. E 84, 015201 (2011).

${ }^{19}$ D. A. Wiley, S. H. Strogatz, and M. Girvan, Chaos 16, 015103 (2006).

${ }^{20}$ T. Gyrnik, private communication (2014).

${ }^{21}$ M. C. Cross and P. C. Hohenberg, Reviews of Modern Physics 65, 851 (1993).

${ }^{22}$ G. Lemoult, L. Shi, K. Avila, S. V. Jalikop, M. Avila, and B. Hof, Nature Physics (2016).

${ }^{23}$ H. Chaté and P. Manneville, Phys. Rev. Lett. 58, 112 (1987).

${ }^{24}$ L. Smirnov, G. Osipov, and A. Pikovsky, Journal of Physics A: Mathematical and Theoretical 50, 08LT01 (2017).

${ }^{25}$ H. Janssen, Z. f. Physik B 42, 151 (1981).

${ }^{26}$ P. Grassberger, Z. f. Physik B 47, 365 (1982).

${ }^{27}$ G. Rousseau, Ph.D. thesis, Université Paris-Diderot-Paris VII (1998).

${ }^{28}$ P. Grassberger and T. Schreiber, Physica D 50, 177 (1991).

${ }^{29}$ T. Bohr, M. van Hecke, R. Mikkelsen, and M. Ipsen, Phys. Rev. Lett. 86, 5482 (2001).

${ }^{30}$ T. Bohr, M. H. Jensen, G. Paladin, and A. Vulpiani, Dynamical systems approach to turbulence (Cambridge University Press, 2005).

${ }^{31}$ K. Kawase and N. Uchida, arXiv:1907.07285 (2019).

${ }^{32}$ P. Jaros, S. Brezetsky, R. Levchenko, D. Dudkowski, T. Kapitaniak, and Y. Maistrenko, Chaos 28, 011103 (2018).

${ }^{33}$ P. Pikovsky and M. Rosenblum, Physica D 238, 27 (2009).

${ }^{34}$ R. Toenjes, N. Masuda, and H. Kori, Chaos 20, 033108 (2010). 
${ }^{35}$ Y. Kawamura, H. Nakao, and Y. Kuramoto, Phys. Rev. E 75, 036209 (2007).

${ }^{36}$ L. Larger, B. Penkovsky, and Y. Maistrenko, Phys. Rev. Lett. 111, 054103 (2013).

${ }^{37}$ S. Olmi, A. Navas, S. Boccaletti, and A. Torcini, Phys. Rev. E 90, 16 (2014).

${ }^{38}$ O. E. Omel'chenko, M. Wolfrum, S. Yanchuk, Y. L. Maistrenko, and O. Sudakov, Phys. Rev. E 85, 036210 (2012).

${ }^{39}$ M. J. Panaggio and D. M. Abrams, Physical review letters 110, 094102 (2013).
${ }^{40}$ J. Xie, E. Knobloch, and H.-C. Kao, Physical Review E 92, 042921 (2015).

${ }^{41}$ Y. Maistrenko, O. Sudakov, O. Osiv, and V. Maistrenko, New Journal of Physics 17, 073037 (2015).

${ }^{42}$ H. W. Lau and J. Davidsen, Phys. Rev. E 94, 010204 (2016).

${ }^{43}$ C. Brito, I. Aranson, and H. Chaté, Phys. Rev. Lett. 90, 068301 (2003).

${ }^{44}$ G. St-Yves and J. Davidsen, J. Chem. Phys. 133, 044909 (2010). 